Published in final edited form as:

Curr Opin Nephrol Hypertens. 2012 May ; 21(3): 334-340. doi:10.1097/MNH.0b013e328351a391.

\title{
Fibroblast Growth Factor 23 and Adverse Clinical Outcomes in Chronic Kidney Disease
}

\author{
Tamara Isakova, MD, MMSc \\ Division of Nephrology and Hypertension, Department of Medicine, University of Miami, Miller \\ School of Medicine, 1120 NW 14th St., Suite 822, Miami, FL 33136, Telephone: 305-243-4374, \\ Fax: 305-243-8914
}

Tamara Isakova: tisakova@med.miami.edu

\section{Abstract}

Purpose of review-To review data on the epidemiology of fibroblast growth factor 23 (FGF23) and adverse clinical outcomes in chronic kidney disease (CKD) and introduce recent insights into the pathophysiology behind the observed relationships.

Recent findings-End-stage renal disease and cardiovascular disease are frequent events in patients with CKD, in whom cardiovascular disease is the leading cause of death. Elevated levels of FGF23, a phosphate and vitamin D regulating hormone, have been associated with risks of endstage renal disease, cardiovascular disease and mortality. FGF23 excess has also been linked with left ventricular hypertrophy, and innovative translational experiments have recently established direct end-organ toxicity of FGF23, which induced left ventricular hypertrophy in animals.

Summary-FGF23 is emerging as a novel risk factor in CKD. Future studies should determine whether interventions that lower FGF23 levels improve clinical outcomes in CKD.

\section{Keywords}

Fibroblast growth factor 23; chronic kidney disease; mortality; cardiovascular disease

\footnotetext{
Disclosures

Dr. Isakova has received honoraria from Genzyme and Shire.

2 stars:

Isakova T, Xie H, Yang W, et al. Fibroblast growth factor 23 and risks of mortality and end-stage renal disease in patients with chronic kidney disease. JAMA 2011; 305: 2432-2439.

** Based on observational data from 3,879 participants with CKD stages 2-3 who enrolled in the CRIC Study, the investigators reported that elevated FGF23 is an independent risk factor for kidney failure and death among CKD patients.

Kendrick J, Cheung AK, Kaufman JS, et al. FGF-23 associates with death, cardiovascular events, and initiation of chronic dialysis. J Am Soc Nephrol 2011; 22: 1913-1922.

** Based on observational data from 1,099 HOST study participants with CKD stages 4-5, the investigators reported that elevated FGF23 is an independent risk factor for cardiovacular events, dialysis initiation, and all-cause mortality.

Faul C, Amaral AP, Oskouei B, et al. FGF23 induces left ventricular hypertrophy. J Clin Invest 2011; In Press.

** This translational report showed that FGF23 caused klotho-independent pathological hypertrophy of isolated rat cardiomyocytes; intramyocardial or intravenous injection of FGF23 in wild-type mice resulted in LVH; klotho-deficient mice demonstrated elevated FGF23 levels and LVH; and in an established animal model of CKD treatment with an FGF-receptor blocker attenuated LVH. These results suggest a causal relationship between FGF23 excess and LVH in CKD.

1 star

Semba RD, Fink JC, Sun K, et al. Serum Fibroblast Growth Factor-23 and Risk of Incident Chronic Kidney Disease in Older Community-Dwelling Women. Clin J Am Soc Nephrol 2011; In Press.

*This observational study reports an independent association between elevated FGF23 levels and incident CKD in elderly women. Moe SM, Zidehsarai MP, Chambers MA, et al. Vegetarian compared with meat dietary protein source and phosphorus homeostasis in chronic kidney disease. Clin J Am Soc Nephrol 2011; 6: 257-264.

*This cross-over study reports that FGF23 levels are significantly decreased after a week of vegetarian diet in participants with CKD.
} 


\section{Introduction}

Chronic kidney disease (CKD) is a common medical problem with recent estimates from the Third National Health and Nutrition Examination Survey suggesting that its prevalence in the US has increased significantly over the last decade [1]. With nearly 23 million Americans affected, exorbitant healthcare costs are consumed in the course of delivering care for this condition. Patients with CKD experience substantial morbidity as they progress to end stage renal disease (ESRD), and mortality rates remain high, with the leading cause of death attributed to cardiovascular disease [2]. While the coexistence of traditional cardiovascular risk factors, such as hypertension and diabetes, in patients with CKD explains some of the excess risk for adverse clinical outcomes, epidemiologic data suggest that the presence of CKD itself may independently confer risk [3,4]. Several possible mechanisms for the consistent association between CKD and cardiovascular disease have been put forth $[5,6]$. The focus of this review is to highlight the evidence in support of the potential role of fibroblast growth factor 23 (FGF23) excess as a mediator of poor outcomes in CKD.

\section{Adverse Clinical Outcomes in CKD}

The presence of CKD, as defined by reduction in estimated glomerular filtration rate (eGFR) alone or in combination with proteinuria, markedly increases risks of kidney failure and cardiovascular mortality $[3,4]$. Prior studies suggest that the rate of cardiovascular disease events in patients with CKD is two to three-fold higher compared to patients without CKD [7], with risk already increasing below eGFR of $60 \mathrm{ml} / \mathrm{min} / 1.73 \mathrm{~m}^{2}$, even more so below eGFR of $45 \mathrm{ml} / \mathrm{min} / 1.73 \mathrm{~m}^{2}$ and a graded relationship over the remaining eGFR spectrum [3]. Rates of CKD progression to ESRD appear to vary by case-mix of study population. While among patients with diabetes and older individuals cardiovascular death is the most common CKD outcome [8,9], progression to ESRD prevails among younger CKD patients $[10,11]$. Moreover, close examination of these and other studies reveals that some patients with CKD maintain stable kidney function over time without experiencing major cardiovascular events. These observations emphasize the urgent need to develop risk stratification strategies in CKD that provide additional information beyond that which is obtained from assessment of eGFR and proteinuria.

\section{Contribution of Established and CKD-specific Risk Factors}

Identification of therapeutic approaches aimed at improving CKD outcomes and reducing costs is another research priority in nephrology. Efforts to address the high prevalence of traditional cardiovascular risk factors, which also contribute to CKD progression, thus far have been met with limited success [12-14]. These disappointing results combined with accumulating literature on the existence of additional CKD-specific risk factors have stimulated prospective investigations of these putative novel risk factors in CKD, with the goal of identifying appropriate interventions for future multi-center clinical trials. The findings for one such risk factor, FGF23 excess, and its associations with intermediate cardiovascular outcomes and hard clinical endpoints are discussed here.

\section{FGF23 and Adverse Clinical Outcomes in CKD}

The discovery and rapid characterization of FGF23 has had important implications for future research direction and perhaps clinical care in CKD. Brief summary of these physiologic findings precedes a review of the recent observational data relating FGF23 to outcomes in CKD. 


\section{Brief Overview of FGF23 Physiology}

FGF23 is secreted by osteocytes and regulates phosphate and vitamin D metabolism [15]. It induces phosphaturia through internalization and degradation of sodium-phosphate cotransporter, NPT2a; inhibits parathyroid hormone secretion; and decreases levels of calcitriol $(1,25 \mathrm{D})$ by inhibiting renal 1-a hydroxylase and stimulating the catabolic $24-$ hydroxylase [16-18]. FGF23 levels increase in health with phosphate loading [19] and in CKD as urinary phosphate excretion declines [20]. In these settings, high FGF23 maintains phosphate balance by increasing phosphate excretion and reducing dietary phosphate absorption via reduced 1,25D [15]. FGF23 levels rise progressively as eGFR declines beginning in CKD stage $2[20,21]$, and levels of the biologically intact hormone can reach 1000 -fold above normal in ESRD [22,23].

\section{Death}

The first epidemiologic study to report on the association between FGF23 and mortality was performed in the dialysis setting. A prospective nested case-control study of 400 incident hemodialysis patients showed that increased FGF23 levels at the initiation of dialysis were independently associated with significantly increased risk of subsequent mortality during the first year on dialysis: individuals with FGF23 levels in the highest quartile were found to be at nearly six times greater adjusted risk of death compared to those with FGF23 levels in the lowest quartile [24]. The results were independent of serum phosphate and demonstrated a strong graded relationship such that ascending quartiles of FGF23 were associated with a linear increase in the risk of mortality (Figure 1).

To date, this initial compelling observation has been confirmed by several other reports, including studies of pre-dialysis CKD, post-kidney transplant and non-CKD patients [2528]. We tested the hypothesis that an elevated FGF23 is independently associated with greater risks of ESRD and mortality in the Chronic Renal Insufficiency Cohort (CRIC) study, which is a prospective, multi-center, observational study of risk factors for CKD progression and cardiovascular disease in CKD patients with baseline eGFR of 20-70 (mean $42.8 \pm 13.5) \mathrm{ml} / \mathrm{min} / 1.73 \mathrm{~m}^{2}[29,30]$. We found that the median FGF23 level among the 3,879 CRIC participants with CKD stages 2-4 was $145 \mathrm{RU} / \mathrm{ml}$ and that $72 \%$ had FGF23 levels above $100 \mathrm{RU} / \mathrm{ml}$ [21], a cut-off previously associated with adverse clinical outcomes in CKD [31]. Moreover, elevated baseline FGF23 was independently associated with greater risk of mortality (Figure 2) over a median follow-up time of 3.5 years, with patients in the highest quartile of FGF23 being at 3-fold greater adjusted risk of death compared to patients in the lowest. Importantly, FGF23 excess was the strongest predictor of mortality, stronger than eGFR and proteinuria, which were not significantly associated with risk of death in the final adjusted models. The robust association persisted across examined eGFR cut-points (Figure 3) [25], and was independent of serum phosphate or parathyroid hormone.

Kendrick et al [26] performed a similar evaluation using stored samples from 1,099 participants in the Homocysteinemia in Kidney and End Stage Renal Disease (HOST) study, a multicenter, randomized, double-blind, placebo-controlled trial of folic acid and B vitamins in a population with advanced CKD [32]. The investigators likewise found a strong independent direct association between baseline FGF23 levels and all-cause mortality among participants with stage 4-5 CKD (mean eGFR of $18 \mathrm{ml} / \mathrm{min} / 1.73 \mathrm{~m}^{2}$; median FGF23 of $392 \mathrm{RU} / \mathrm{ml}$ ), such that individuals in the highest quartile of FGF23 had a 2.2-fold greater adjusted risk of death than patients in the lowest quartile during a median follow-up of 2.9 years. Notably, adjustment for other markers of mineral metabolism, including serum calcium, phosphate, parathyroid hormone and vitamin D levels, did not alter these findings. 


\section{Kidney Disease Progression}

Two small studies have previously suggested that elevated FGF23 could be an independent risk factor for more rapid loss of kidney function in CKD [31,33]. One was a prospective study of 177 non-diabetic CKD patients, and it examined the association between FGF23 levels and kidney disease progression defined as doubling of serum creatinine and/or terminal kidney failure. During a median follow up of 53 months, FGF23 levels > 104 RU/ $\mathrm{ml}$ compared to lower levels were associated with more rapid progression of CKD, independent of age, gender, baseline eGFR, proteinuria, and serum levels of calcium, phosphate, and parathyroid hormone [31]. The second study was conducted in 55 patients with diabetic nephropathy, in whom elevated FGF23 was associated with the composite primary outcome of death, doubling of serum creatinine, or dialysis [33]. These early findings were recently supported by the observations in the CRIC study, in which elevated FGF23 was an independent risk factor for ESRD in patients with relatively preserved kidney function (eGFR $230 \mathrm{ml} / \mathrm{min} / 1.73 \mathrm{~m}^{2}$ ), but not in those with eGFR $<30 \mathrm{ml} / \mathrm{min} / 1.73 \mathrm{~m}^{2}$ [25]. Moreover, in the HOST study elevated FGF23 levels were independently associated with risk of chronic dialysis initiation [26]. Notably, several independent reports show that FGF23 levels correlate with proteinuria [25,33] in CKD. Finally, a recent study of 701 older women suggested that elevated FGF23 may independently predict the onset of incident CKD, though the study was limited by use of the modified Modification of Diet in Renal Disease estimating equation to define CKD in the elderly population [34].

\section{Cardiovascular Disease}

FGF23 appears to be a risk factor for cardiovascular disease events in CKD, as has been demonstrated in a study of 149 CKD patients, in whom an elevated baseline FGF23 level predicted subsequent myocardial infarction, coronary, carotid and lower limb artery interventions, non-traumatic lower extremity amputation, or death [35]. In the secondary analysis of 1099 HOST study participants Kendrick et al also found a strong relationship between higher FGF23 levels and greater risks of acute myocardial infarction and lower extremity limb amputations [26]. Similarly, in a cohort of 833 patients with stable coronary artery disease but low prevalence of decreased eGFR, elevated FGF23 predicted future risk of a composite of cardiovascular disease events that included myocardial infarction, stroke, transient ischemic attack, and heart failure [27]. Importantly, when the components of the composite were considered individually, the authors found significant associations between doubling of FGF23 levels and risks of heart failure and stroke or transient ischemic attack, but not risk of myocardial infarction. Likewise, a recent report of a nested case-control study in the Health Professionals Follow-up Study found no significant association between baseline FGF23 levels and future risk of nonfatal myocardial infarction and fatal coronary heart disease [36]. Notably, congestive heart failure and stroke were not evaluated in this latter study. Whether these disparate findings point to specificity of FGF23 effect requires further investigation. However, the association of FGF23 with markers of myocardial injury (brain natriuretic peptide [37] and troponin [38,39]) and with left ventricular hypertrophy (as described below) potentially point to such targeted toxicity of FGF23.

\section{Left Ventricular Hypertrophy}

Several recent studies support a relationship between FGF23 and left ventricular hypertrophy (LVH). In the first study of 124 prevalent hemodialysis patients, higher FGF23 but not serum phosphate levels were independently associated with LVH and increased left ventricular mass index (LVMI) [40]. A second study showed that elevated FGF23 levels were associated with increased LVMI and increased prevalence of LVH in 162 pre-dialysis CKD patients, independent of traditional risk factors and serum phosphate levels, which were not associated with LVMI or LVH [41]. Other recent studies have confirmed initial reports $[37,42-45]$. The largest of these was performed in the CRIC study, in which 3070 
stage 2-4 CKD patients had baseline FGF23 measurements and underwent echocardiograms one year later [45]. Consistent with prior reports, the investigators showed that ascending quartiles of FGF23 were significantly associated with increased LVMI and greater prevalence of concentric and eccentric LVH, independent of demographic, clinical and laboratory covariates, including eGFR, hemoglobin, albuminuria, parathyroid hormone, and serum phosphate. Importantly, the investigators were also able to prospectively examine the association between elevated FGF23 and incident LVH in 411 CRIC participants who had normal left ventricular geometry on their baseline echocardiogram and had follow-up echocardiograms available at a subsequent study visit. This analysis showed that elevated FGF23 levels at baseline were associated with increased future risk of incident LVH, supporting a possible causal link between FGF23 excess and LVH, which was confirmed in animal and in vitro experiments (see below).

\section{Vascular Calcification}

The results for vascular calcification are conflicting. While several investigators were not able to find a significant relationship between FGF23 and vascular calcification measured radiographically in individuals with healthy kidney function [46], dialysis patients [47], and patients with earlier stages of CKD [41], others did report an association with coronary calcification scores in the dialysis setting [48] and with other measures of vascular disease in the general population and in those with reduced eGFR [49,50]. For example, higher FGF23 levels were independently associated with impaired vasoreactivity in 759 patients with normal kidney function and with arterial stiffness in 208 patients with eGFR $<60 \mathrm{~mL} / \mathrm{min} /$ $1.73 \mathrm{~m}^{2}$ [50]. Most recently, another report of 142 patients with CKD stages 2-5, including patients on dialysis, found that elevated FGF23 levels were associated with higher aortic calcification scores independent of CKD stage and age [51]. Larger studies are needed to confirm or refute these findings.

\section{Pathophysiology of FGF23 Toxicity}

Elevated FGF23 is independently associated with LVH in CKD, but a causal relationship cannot be established with observational studies. To bridge this gap, Faul et al performed a series of translational studies to determine whether FGF23 contributes directly or is simply a biomarker of toxicity of other pathogenetic factors that are important in CKD [45]. First, the investigators showed that FGF23 causes pathological hypertrophy of isolated cardiac myocytes in vitro via FGF receptor-dependent activation but independent of klotho, the coreceptor for FGF23 in the kidney and parathyroid glands. Second, intra-myocardial or intravenous injection of FGF23 in wild-type mice resulted in LVH, and klotho deficient and klotho heterozygous mice demonstrated both elevated FGF23 levels and LVH in a genedose-dependent pattern. Finally, administering an FGF receptor blocker to the 5/6 nephrectomy rat model of CKD that is known to develop LVH, severe hypertension, and elevated FGF23, attenuated LVH without reduction in blood pressure or FGF23. Taken together, these results provide the first evidence of a klotho-independent, causal role for FGF23 in the pathogenesis of LVH, and suggest that chronically elevated FGF23 levels may contribute directly to high prevalence of LVH in CKD. Given that cardiovascular disease is the primary cause of death in CKD, and LVH is an important mechanism of this relationship [52,53], these experimental findings provide support for a causal relationship between FGF23 and mortality in this population.

\section{Implications for Therapy}

The identification of elevated FGF23 as a potent risk factor and potential mechanism of adverse outcomes in CKD emphasizes the critical need to investigate therapeutic strategies to lower FGF23. Early physiologic studies performed in healthy volunteers suggested that 
FGF23 levels may be modifiable with use of dietary phosphate restriction and phosphate binders [19,54]. Clinical data regarding the impact of binders on FGF23 levels in dialysis and in stage 3-4 CKD is beginning to accumulate [55-57]. For example, following four weeks of treatment with sevelamer hydrochloride and calcium carbonate FGF23 levels decreased significantly from pretreatment levels in patients undergoing dialysis [58]. Oliveira et al showed that FGF23 levels decreased by 50\% in normophosphatemic patients with CKD stages 3-4 following six weeks of sevelamer hydrochloride therapy [55]. Similarly, use of lanthanum carbonate for four weeks resulted in $22 \%$ reduction in FGF23 levels in patients with stage $3 \mathrm{CKD}$ and baseline serum phosphate $<4.5 \mathrm{mg} / \mathrm{dl}$ [56]. There appears to be a hint of lack of efficacy on FGF23 lowering with calcium-based binders in both the dialysis setting [58] and in CKD [55], but larger studies are needed to verify this early signal. Finally, two weeks of lanthanum carbonate therapy in a pilot study of normophosphatemic stage 3-4 CKD did not result in change in FGF23 [57], suggesting that studies of longer duration are needed to manifest efficacy. While data on dietary interventions are less readily available, a recent a crossover trial in nine patients with a mean eGFR of $32 \mathrm{ml} / \mathrm{min}$ showed that one week of vegetarian diet significantly lowered FGF23 levels [59]. Additional therapeutic approaches might emerge from further investigation of the specificity of the cardiac FGF receptors, as suggested by Faul et al [45], and from ongoing clinical studies of the efficacy and safety of FGF23 antibodies in the treatment of X-linked hypophosphatemia [60].

\section{Conclusions}

A substantial body of observational data has now established elevated FGF23 as a strong independent risk factor for adverse outcomes in CKD. This predictive ability positions it as a novel biomarker that could help identify which of the millions of patients who are newly diagnosed with CKD will progress to ESRD or die prematurely. Moreover, emerging data on the toxic effects of FGF23 on the myocardium suggest that FGF23 excess in CKD is a novel therapeutic target worthy of further experimental work. Human studies should evaluate the efficacy of existing and novel treatments to safely lower FGF23 levels and the resultant effects on intermediate cardiovascular and renal outcomes. Randomized clinical trials will then be needed to determine whether FGF23 lowering strategies will improve hard clinical end points in CKD.

\section{Acknowledgments}

Dr. Isakova was supported by grant K23DK087858 from the National Institutes of Health.

\section{References}

1. Coresh J, Selvin E, Stevens LA, et al. Prevalence of chronic kidney disease in the United States. JAMA. 2007; 298:2038-2047. [PubMed: 17986697]

2. US Renal Data System. USRDS 2010 Annual Data Report: Atlas of Chronic Kidney Disease and End-Stage Renal Disease in the United States. National Institutes of Health, National Institute of Diabetes and Digestive and Kidney Diseases; Bethesda, MD: 2010.

3. Go AS, Chertow GM, Fan D, et al. Chronic kidney disease and the risks of death, cardiovascular events, and hospitalization. N Engl J Med. 2004; 351:1296-1305. [PubMed: 15385656]

4. Hemmelgarn BR, Manns BJ, Lloyd A, et al. Relation between kidney function, proteinuria, and adverse outcomes. JAMA. 2010; 303:423-439. [PubMed: 20124537]

5. Sarnak MJ, Levey AS, Schoolwerth AC, et al. Kidney disease as a risk factor for development of cardiovascular disease: a statement from the American Heart Association Councils on Kidney in Cardiovascular Disease, High Blood Pressure Research, Clinical Cardiology, and Epidemiology and Prevention. Circulation. 2003; 108:2154-2169. [PubMed: 14581387] 
6. Go AS, Lo JC. Epidemiology of non-dialysis-requiring chronic kidney disease and cardiovascular disease. Curr Opin Nephrol Hypertens. 2006; 15:296-302. [PubMed: 16609298]

7. Shulman NB, Ford CE, Hall WD, et al. Prognostic value of serum creatinine and effect of treatment of hypertension on renal function. Results from the hypertension detection and follow-up program. The Hypertension Detection and Follow-up Program Cooperative Group. Hypertension. 1989; 13:I80-93. [PubMed: 2490833]

8. Adler AI, Stevens RJ, Manley SE, et al. Development and progression of nephropathy in type 2 diabetes: the United Kingdom Prospective Diabetes Study (UKPDS 64). Kidney Int. 2003; 63:225232. [PubMed: 12472787]

9. Dalrymple LS, Katz R, Kestenbaum B, et al. Chronic kidney disease and the risk of end-stage renal disease versus death. J Gen Intern Med. 2011; 26:379-385. [PubMed: 20853156]

10. Levin A, Djurdjev O, Beaulieu M, et al. Variability and risk factors for kidney disease progression and death following attainment of stage 4 CKD in a referred cohort. Am J Kidney Dis. 2008; 52:661-671. [PubMed: 18805347]

11. Landray MJ, Emberson JR, Blackwell L, et al. Prediction of ESRD and death among people with CKD: the Chronic Renal Impairment in Birmingham (CRIB) prospective cohort study. Am J Kidney Dis. 2010; 56:1082-1094. [PubMed: 21035932]

12. Appel LJ, Wright JT, Greene T, et al. Intensive blood-pressure control in hypertensive chronic kidney disease. N Engl J Med. 2010; 363:918-929. [PubMed: 20818902]

13. de Boer IH, Rue TC, Hall YN, et al. Temporal trends in the prevalence of diabetic kidney disease in the United States. JAMA. 2011; 305:2532-2539. [PubMed: 21693741]

14. Baigent C, Landray MJ, Reith C, et al. The effects of lowering LDL cholesterol with simvastatin plus ezetimibe in patients with chronic kidney disease (Study of Heart and Renal Protection): a randomised placebo-controlled trial. Lancet. 2011; 377:2181-2192. [PubMed: 21663949]

15. Wolf M. Forging forward with 10 burning questions on FGF23 in kidney disease. J Am Soc Nephrol. 2010; 21:1427-1435. [PubMed: 20507943]

16. Shimada T, Hasegawa H, Yamazaki Y, et al. FGF-23 is a potent regulator of vitamin D metabolism and phosphate homeostasis. J Bone Miner Res. 2004; 19:429-435. [PubMed: 15040831]

17. Shimada T, Kakitani M, Yamazaki Y, et al. Targeted ablation of Fgf23 demonstrates an essential physiological role of FGF23 in phosphate and vitamin D metabolism. J Clin Invest. 2004; 113:561-568. [PubMed: 14966565]

18. Ben-Dov IZ, Galitzer H, Lavi-Moshayoff V, et al. The parathyroid is a target organ for FGF23 in rats. J Clin Invest. 2007; 117:4003-4008. [PubMed: 17992255]

19. Ferrari SL, Bonjour J-P, Rizzoli R. Fibroblast growth factor-23 relationship to dietary phosphate and renal phosphate handling in healthy young men. J Clin Endocrinol Metab. 2005; 90:15191524. [PubMed: 15613425]

20. Gutierrez O, Isakova T, Rhee E, et al. Fibroblast growth factor-23 mitigates hyperphosphatemia but accentuates calcitriol deficiency in chronic kidney disease. J Am Soc Nephrol. 2005; 16:22052215. [PubMed: 15917335]

21. Isakova T, Wahl P, Vargas GS, et al. Fibroblast growth factor 23 is elevated before parathyroid hormone and phosphate in chronic kidney disease. Kidney Int. 2011; 79:1370-1378. [PubMed: 21389978]

22. Isakova T, Gutierrez OM, Wolf M. A blueprint for randomized trials targeting phosphorus metabolism in chronic kidney disease. Kidney Int. 2009; 76:705-716. [PubMed: 19606082]

23. Shimada T, Urakawa I, Isakova T, et al. Circulating fibroblast growth factor 23 in patients with end-stage renal disease treated by peritoneal dialysis is intact and biologically active. J Clin Endocrinol Metab. 2010; 95:578-585. [PubMed: 19965919]

24. Gutierrez OM, Mannstadt M, Isakova T, et al. Fibroblast growth factor 23 and mortality among patients undergoing hemodialysis. N Engl J Med. 2008; 359:584-592. [PubMed: 18687639]

25. Isakova T, Xie H, Yang W, et al. Fibroblast growth factor 23 and risks of mortality and end-stage renal disease in patients with chronic kidney disease. JAMA. 2011; 305:2432-2439. [PubMed: 21673295]

26. Kendrick J, Cheung AK, Kaufman JS, et al. FGF-23 associates with death, cardiovascular events, and initiation of chronic dialysis. J Am Soc Nephrol. 2011; 22:1913-1922. [PubMed: 21903574] 
27. Parker BD, Schurgers LJ, Brandenburg VM, et al. The associations of fibroblast growth factor 23 and uncarboxylated matrix Gla protein with mortality in coronary artery disease: the Heart and Soul Study. Ann Intern Med. 2010; 152:640-648. [PubMed: 20479029]

28. Wolf M, Molnar MZ, Amaral AP, et al. Elevated fibroblast growth factor 23 is a risk factor for kidney transplant loss and mortality. J Am Soc Nephrol. 2011; 22:956-966. [PubMed: 21436289]

29. Feldman HI, Appel LJ, Chertow GM, et al. The Chronic Renal Insufficiency Cohort (CRIC) Study: Design and Methods. J Am Soc Nephrol. 2003; 14:S148-153. [PubMed: 12819321]

30. Fischer MJ, Go AS, Lora CM, et al. CKD in Hispanics: Baseline characteristics from the CRIC (Chronic Renal Insufficiency Cohort) and Hispanic-CRIC Studies. Am J Kidney Dis. 2011; 58:214-227. [PubMed: 21705121]

31. Fliser D, Kollerits B, Neyer U, et al. Fibroblast growth factor 23 (FGF23) predicts progression of chronic kidney disease: the Mild to Moderate Kidney Disease (MMKD) Study. J Am Soc Nephrol. 2007; 18:2600-2608. [PubMed: 17656479]

32. Jamison RL, Hartigan P, Kaufman JS, et al. Effect of homocysteine lowering on mortality and vascular disease in advanced chronic kidney disease and end-stage renal disease: a randomized controlled trial. JAMA. 2007; 298:1163-1170. [PubMed: 17848650]

33. Titan SM, Zatz R, Graciolli FG, et al. FGF-23 as a predictor of renal outcome in diabetic nephropathy. Clin J Am Soc Nephrol. 2011; 6:241-247. [PubMed: 20966122]

34. Semba RD, Fink JC, Sun K, et al. Serum fibroblast growth factor-23 and risk of incident chronic kidney disease in older community-dwelling women. Clin J Am Soc Nephrol. 2011 In Press.

35. Seiler S, Reichart B, Roth D, et al. FGF-23 and future cardiovascular events in patients with chronic kidney disease before initiation of dialysis treatment. Nephrol Dial Transplant. 2010; 25:3983-3989. [PubMed: 20525642]

36. Taylor EN, Rimm EB, Stampfer MJ, et al. Plasma fibroblast growth factor 23, parathyroid hormone, phosphorus, and risk of coronary heart disease. Am Heart J. 2011; 161:956-962. [PubMed: 21570529]

37. Seiler S, Cremers B, Rebling NM, et al. The phosphatonin fibroblast growth factor 23 links calcium-phosphate metabolism with left-ventricular dysfunction and atrial fibrillation. Eur Heart J. 2011; 32:2688-2696. [PubMed: 21733911]

38. Ford ML, Smith ER, Tomlinson LA, et al. FGF-23 and osteoprotegerin are independently associated with myocardial damage in chronic kidney disease stages 3 and 4 . Another link between chronic kidney disease-mineral bone disorder and the heart. Nephrol Dial Transplant. 2011 In Press.

39. Holden RM, Beseau D, Booth SL, et al. FGF-23 is associated with cardiac troponin T and mortality in hemodialysis patients. Hemodial Int. 2011 In Press.

40. Hsu HJ, Wu MS. Fibroblast growth factor 23: a possible cause of left ventricular hypertrophy in hemodialysis patients. Am J Med Sci. 2009; 337:116-122. [PubMed: 19214027]

41. Gutierrez OM, Januzzi JL, Isakova T, et al. Fibroblast growth factor 23 and left ventricular hypertrophy in chronic kidney disease. Circulation. 2009; 119:2545-2552. [PubMed: 19414634]

42. Mirza MA, Larsson A, Melhus H, et al. Serum intact FGF23 associate with left ventricular mass, hypertrophy and geometry in an elderly population. Atherosclerosis. 2009; 207:546-551. [PubMed: 19524924]

43. Canziani ME, Tomiyama C, Higa A, et al. Fibroblast growth factor 23 in chronic kidney disease: bridging the gap between bone mineral metabolism and left ventricular hypertrophy. Blood Purif. 2011; 31:26-32. [PubMed: 21135546]

44. Kirkpantur A, Balci M, Gurbuz OA, et al. Serum fibroblast growth factor-23 (FGF-23) levels are independently associated with left ventricular mass and myocardial performance index in maintenance haemodialysis patients. Nephrol Dial Transplant. 2011; 26:1346-1354. [PubMed: 20813767]

45. Faul C, Amaral AP, Oskouei B, et al. FGF23 induces left ventricular hypertrophy. J Clin Invest. 2011 In Press.

46. Roos M, Lutz J, Salmhofer H, et al. Relation between plasma fibroblast growth factor-23, serum fetuin-A levels and coronary artery calcification evaluated by multislice computed tomography in 
patients with normal kidney function. Clin Endocrinol (Oxf). 2008; 68:660-665. [PubMed: 17970775]

47. Inaba M, Okuno S, Imanishi $\mathrm{Y}$, et al. Role of fibroblast growth factor-23 in peripheral vascular calcification in non-diabetic and diabetic hemodialysis patients. Osteoporos Int. 2006; 17:15061513. [PubMed: 16896512]

48. Srivaths PR, Goldstein SL, Silverstein DM, et al. Elevated FGF 23 and phosphorus are associated with coronary calcification in hemodialysis patients. Pediatr Nephrol. 2011; 26:945-951.

[PubMed: 21359960]

49. Mirza MA, Hansen T, Johansson L, et al. Relationship between circulating FGF23 and total body atherosclerosis in the community. Nephrol Dial Transplant. 2009; 24:3125-3131. [PubMed: 19429932]

50. Mirza MA, Larsson A, Lind L, et al. Circulating fibroblast growth factor-23 is associated with vascular dysfunction in the community. Atherosclerosis. 2009; 205:385-390. [PubMed: 19181315]

51. Desjardins L, Liabeuf S, Renard C, et al. FGF23 is independently associated with vascular calcification but not bone mineral density in patients at various CKD stages. Osteoporos Int. 2011 In Press.

52. Silberberg JS, Barre PE, Prichard SS, et al. Impact of left ventricular hypertrophy on survival in end-stage renal disease. Kidney Int. 1989; 36:286-290. [PubMed: 2528654]

53. Levy D, Garrison RJ, Savage DD, et al. Prognostic implications of echocardiographically determined left ventricular mass in the Framingham Heart Study. N Engl J Med. 1990; 322:15611566. [PubMed: 2139921]

54. Burnett S, Gunawardene S, Bringhurst F, et al. Regulation of C-terminal and intact FGF-23 by dietary phosphate in men and women. J Bone Miner Res. 2006; 21:1187-1196. [PubMed: 16869716]

55. Oliveira RB, Cancela AL, Graciolli FG, et al. Early control of PTH and FGF23 in normophosphatemic CKD patients: a new target in CKD-MBD therapy? Clin J Am Soc Nephrol. 2010; 5:286-291. [PubMed: 19965540]

56. Gonzalez-Parra E, Gonzalez-Casaus ML, Galan A, et al. Lanthanum carbonate reduces FGF23 in chronic kidney disease Stage 3 patients. Nephrol Dial Transplant. 2011; 26:2567-2571. [PubMed: 21436379]

57. Isakova T, Gutierrez OM, Smith K, et al. Pilot study of dietary phosphorus restriction and phosphorus binders to target fibroblast growth factor 23 in patients with chronic kidney disease. Nephrol Dial Transplant. 2011; 26:584-591. [PubMed: 20631407]

58. Koiwa F, Kazama JJ, Tokumoto A, et al. Sevelamer hydrochloride and calcium bicarbonate reduce serum fibroblast growth factor 23 levels in dialysis patients. Ther Apher Dial. 2005; 9:336-339. [PubMed: 16076378]

59. Moe SM, Zidehsarai MP, Chambers MA, et al. Vegetarian compared with meat dietary protein source and phosphorus homeostasis in chronic kidney disease. Clin J Am Soc Nephrol. 2011; 6:257-264. [PubMed: 21183586]

60. Aono Y, Yamazaki Y, Yasutake J, et al. Therapeutic effects of anti-FGF23 antibodies in hypophosphatemic rickets/osteomalacia. J Bone Miner Res. 2009; 24:1879-1888. [PubMed: 19419316] 


\section{Key points}

- FGF23 is elevated in most CKD stage 2-4 patients (>10 million in US).

- Elevated FGF23 is an independent risk factor for ESRD and death in the CKD population.

- Elevated FGF23 is independently associated with incident and prevalent LVH.

- FGF23 exerts hypertrophic effects on the heart, suggesting a causal role for FGF23 in the pathogenesis of LVH. 


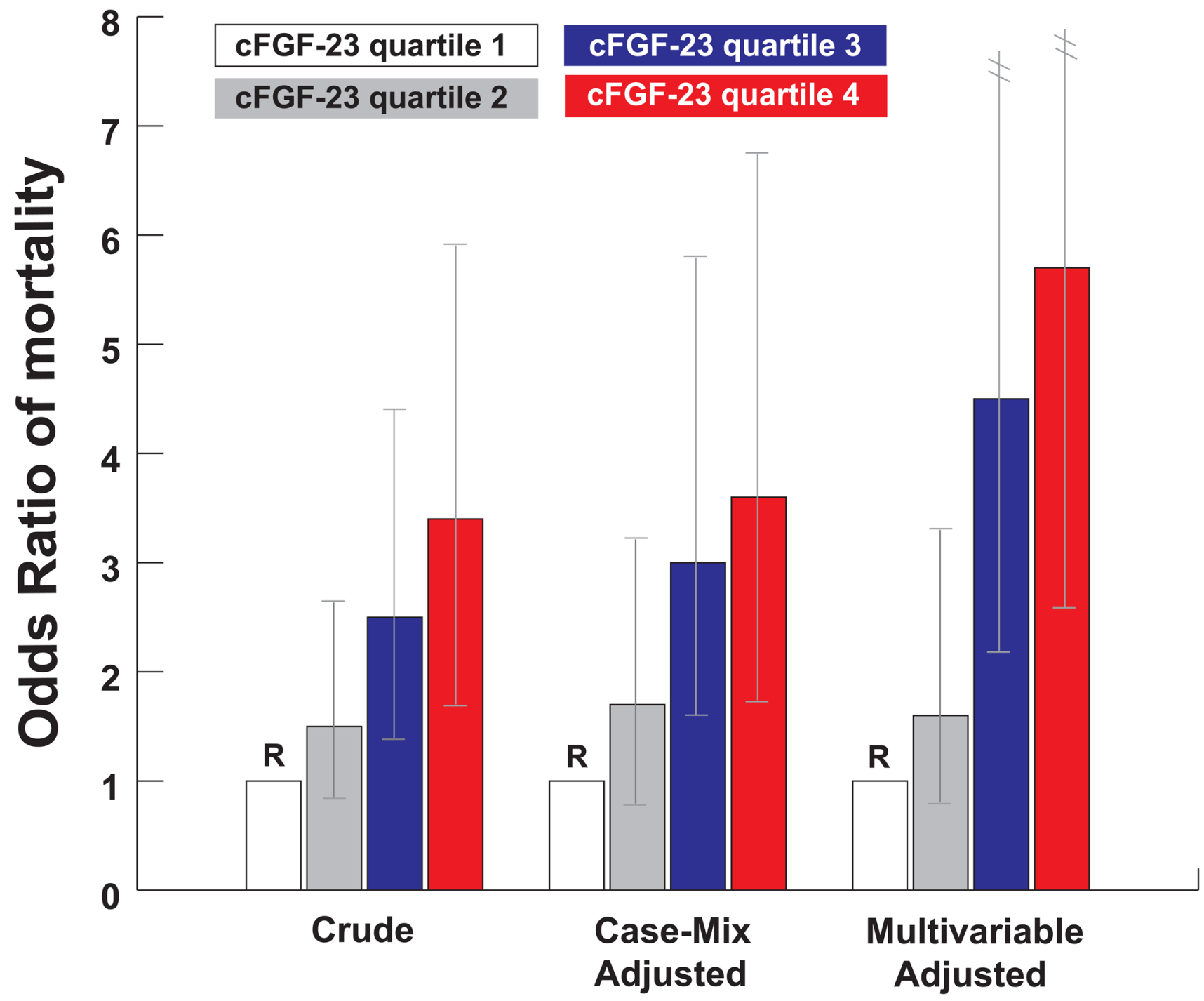

Figure 1. Crude, case-mix-adjusted, and multivariable-adjusted odds ratio of mortality according to quartiles of cFGF23

The case-mix-adjusted analysis included the following variables: age, sex, race, ethnicity, blood pressure, body mass index, standardized mortality rate, vascular access, history of diabetes, and congestive heart failure. The multivariable-adjusted analysis included the casemix variables plus phosphate, calcium, log parathyroid hormone, albumin, creatinine, and ferritin. Quartile 1 is the reference group in all models (Quartile 1, < $1090 \mathrm{RU} / \mathrm{ml}$; Quartile 2, 1090-1750 RU/ml; Quartile 3, 1751-4010 RU/ml; Quartile 4, > 4010 RU/ml). Vertical lines represent $95 \%$ confidence intervals. This figure is reproduced from Gutiérrez et al [24], with permission from the Massachusetts Medical Society. Copyright c [2008] Massachusetts Medical Society. All rights reserved. 


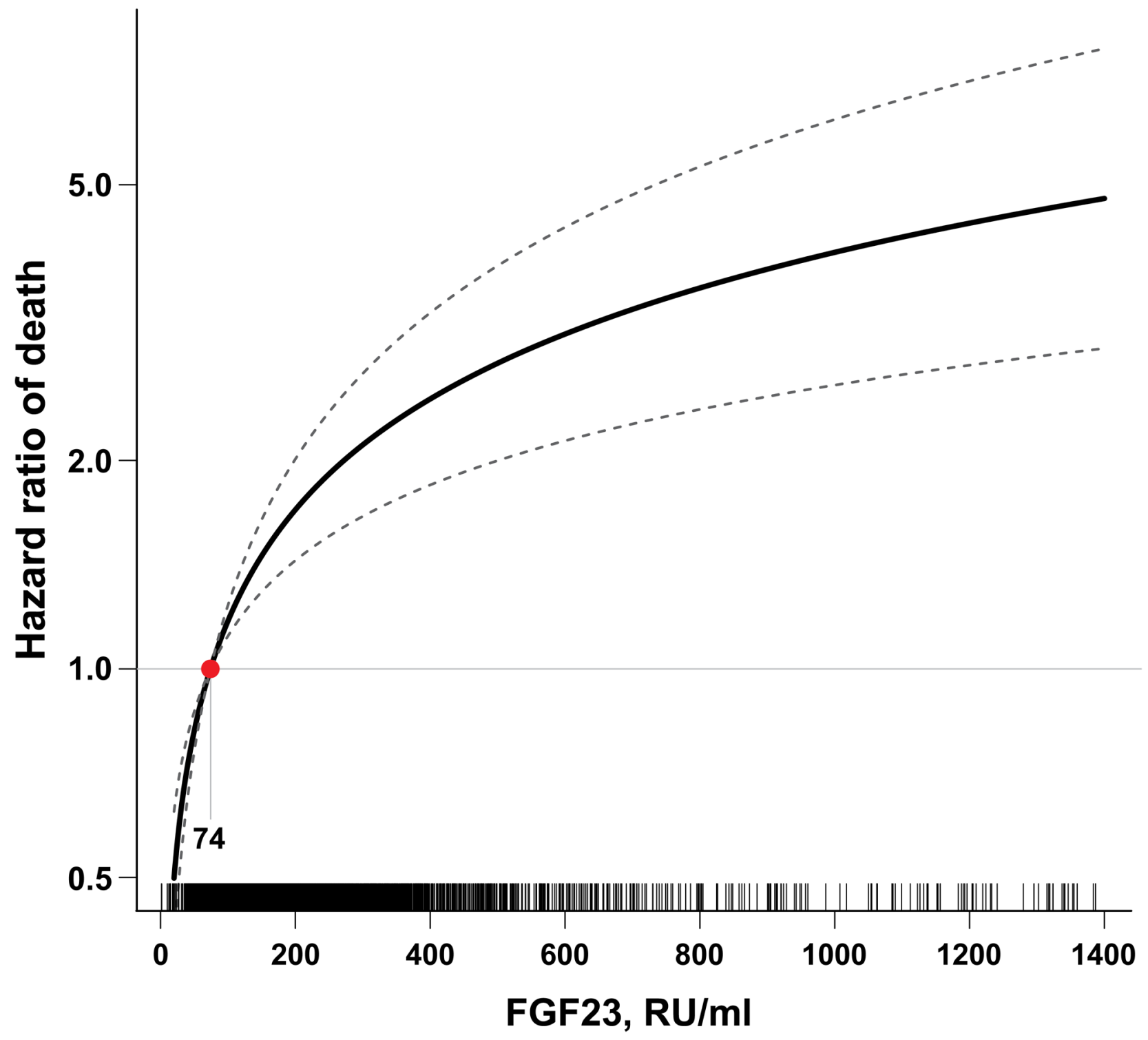

Figure 2. Multivariable-adjusted hazard function for death according to FGF23 levels The median FGF23 level within the lowest FGF23 quartile (74 RU/ml) served as the referent value (hazard $=1$ ). The model was stratified by center and adjusted for age, sex, race, ethnicity, estimated glomerular filtration rate, natural log-transformed urine albuminto-creatinine ratio, hemoglobin, serum albumin, systolic blood pressure, body mass index, diabetes, smoking, low density lipoprotein, history of coronary artery disease, congestive heart failure, stroke, and peripheral vascular disease, and use of aspirin, beta-blockers, statins, and angiotensin converting enzyme inhibitors or angiotensin receptor blockers, and serum calcium, phosphate and natural log-transformed parathyroid hormone. Tick marks on the $\mathrm{x}$ axis indicate individual observations at corresponding levels of FGF23. This figure is reproduced from Isakova et al [25], with permission from the American Medical Association. Copyright c [2011] American Medical Association. All rights reserved. 
Risk of ESRD

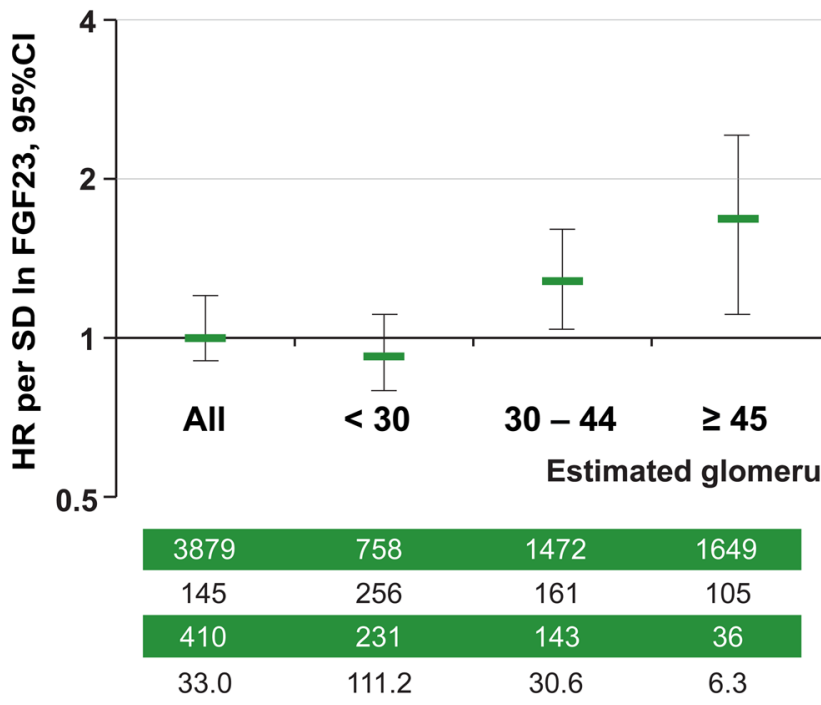

Risk of Death

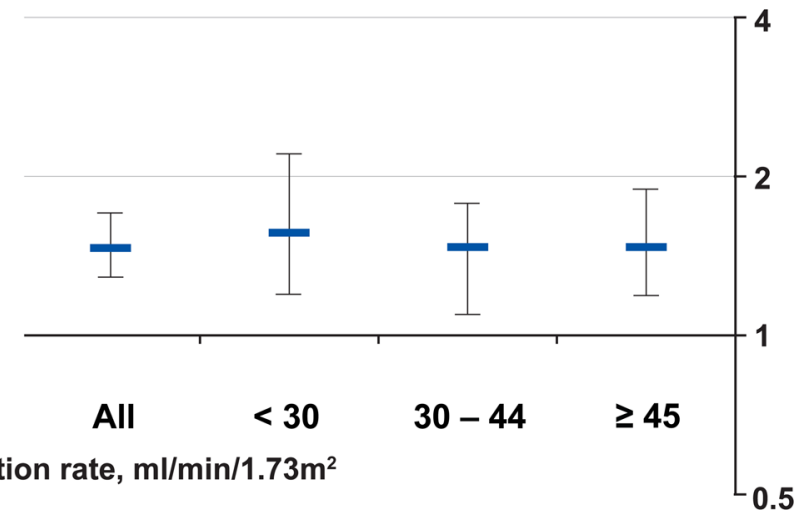

\begin{tabular}{ccccc} 
No. & 3879 & 758 & 1472 & 1649 \\
\hline FGF23 & 145 & 256 & 161 & 105 \\
Events & 266 & 79 & 108 & 79 \\
\hline ncidence & 20.3 & 31.6 & 22.0 & 13.8
\end{tabular}

Figure 3. FGF23 levels and risks of ESRD and death according to baseline kidney function Multivariable-adjusted risks of ESRD and death per unit increment in standard deviation (SD) of natural log-transformed (ln) FGF23 in all participants and according to categories of baseline estimated glomerular filtration rate (eGFR). Models were stratified by center and adjusted for age, sex, race, ethnicity, natural log-transformed urine albumin-to-creatinine ratio, hemoglobin, serum albumin, systolic blood pressure, body mass index, diabetes, smoking, low density lipoprotein, history of coronary artery disease, congestive heart failure, stroke, and peripheral vascular disease, and use of aspirin, beta-blockers, statins, and angiotensin converting enzyme inhibitors or angiotensin receptor blockers, and serum calcium, phosphate and natural log-transformed parathyroid hormone. Error-bars indicate 95\% confidence intervals. The number of participants (No.), their median FGF23 levels, total number of events, and the unadjusted event rate, expressed per 1000 person-years, are presented for the categories of baseline eGFR. Abbreviations: ESRD, end-stage renal disease; HR, hazard ratio; SD, standard deviation. This figure is reproduced from Isakova et al [25], with permission from the American Medical Association. Copyright c [2011] American Medical Association. All rights reserved. 